\title{
El activismo digital desde la mirada de las ONG y las personas jóvenes
}

\author{
Juan Sebastián Fernández-Prados' ${ }^{1}$, Carlos Alonso Carmona², María \\ Teresa Martín Palomo ${ }^{1}$ y Jesús Muyor-Rodríguez ${ }^{3}$ \\ 1 Departamento de Geografía, Historia y Humanidades, CEMyRI, Universidad de Almería \\ España | jsprados@ual.es; tmartinp@ual.es | https://orcid.org/0000-0002-7419-3998; \\ https://orcid.org/0000-0002-0476-6543 \\ 2 Departamento de Sociología, Universidad Pablo de Olavide, Sevilla España \\ calonsocarmona@gmail.com | http://orcid.org/0000-0003-3322-2307 \\ ${ }^{3}$ Departamento de Psicología, Universidad de Almería, España | jesusmuyor@ual.es | \\ https://orcid.org/0000-0003-4226-5181
}

\begin{abstract}
Resumen: Los recursos digitales se han convertido en un elemento clave para entender los nuevos procesos de participación ciudadana. En los últimos años se ha intensificado el empleo de las nuevas tecnologías digitales para relación entre la ciudadanía y los decisores públicos, así como con diversos movimientos sociales y proyectos activistas. La capacidad de acción de las organizaciones de la sociedad civil está cada vez más ligada a sus estrategias comunicativas en el medio online, especialmente cuando se trata de atraer e implicar a las generaciones más jóvenes. El objetivo de esta investigación es explorar qué elementos facilitan o dificultan la participación digital de las y los jóvenes andaluces en un sector específico de organizaciones: las entidades del Tercer Sector de Acción Social que operan en su comunidad autónoma. Para analizarlo, recurrimos a un abordaje cualitativo combinando dos técnicas.. Examinamos la adaptación a las nuevas tecnologías de la comunicación y las estrategias en redes de una muestra de entidades, por medio de entrevistas semiestructuradas a responsables de comunicación de dichas organizaciones. Por otro lado, analizamos el discurso del público potencial de sus estrategias comunicativas, mediante grupos de discusión con jóvenes con experiencias ciberactivistas y perfiles activos en redes sociales. Los resultados muestran diversos obstáculos a la existencia de una participación digital efectiva. Por parte de las entidades, se observa una tendencia a la priorización del "trabajo de calle" frente a las estrategias en red, que siguen entendiéndose como un ámbito acotado a los profesionales de la comunicación, y no como un elemento transversal a la actividad de la entidad. Por parte de las y los jóvenes activistas, existe la percepción de este tipo de entidades como excesivamente institucionalizadas y con demandas alejadas de sus problemáticas cotidianas.
\end{abstract}

Palabras clave: Participación Digital; Jóvenes; Tercer Sector; Redes Sociales; Investigación Cualitativa.

\section{Digital Activism from the Perspective of NGO and Young People}

\begin{abstract}
Digital resources have become a key element in understanding new processes of citizen participation. In recent years, the use of new digital technologies has intensified in the relationship between citizens and public decision-makers, as well as with various social movements and activist projects. The ability for action of civil society organisations is increasingly linked to their communication strategies in the online environment, especially when it comes to attracting and involving the younger generations. The aim of this research is to understand what elements ease or hinder the smooth digital participation of young Andalusians in a specific sector of organisations: The Third Sector Social Action entities using in their autonomous community. To analyse this, we resorted to a combination of several qualitative techniques. We examined the adaptation to new communication technologies and network strategies of a sample of organisations by means of in-depth interviews with those responsible for communication in these organisations. On the other hand, we analysed the discourse of the potential audience of their communication strategies, through discussion groups with young people with cyberactivist experiences and active profiles in social networks. The results show various obstacles to the existence of effective digital participation. On the part of the organisations, there is a tendency to prioritise "street work" over online strategies, which continue to be understood as a field limited to communication professionals, and not as a transversal element in the organisation's activity. Young activists perceive this type of organisation as excessively institutionalised and with demands that are far removed from their day-to-day problems.
\end{abstract}

Keywords: Digital Participation; Youth; Third Sector; Social Networks; Qualitative Research. 


\section{Introducción}

La participación social y política en el contexto de la sociedad digital se ha convertido en un tema actual y relevante para investigadores y decisores públicos (Castells, 2012, Ortiz, 2016). Su importancia se intensifica en el actual contexto de pandemia global, en el cual las formas de participación tradicionales ligadas a la presencialidad se han visto severamente restringidas. En estas circunstancias, resulta de interés responder a la siguiente pregunta examinar qué elementos dificultan o facilitan la implicación digital de la ciudadanía en el mundo asociativo. En concreto, tratamos de responder a la siguiente pregunta ¿qué obstáculos encuentran las entidades del Tercer Sector de Acción social en Andalucía a la hora de fomentar la participación digital de las y los jóvenes?

Esta investigación trata de ofrecer algunas claves para entender la participación social por vías digitales de un sector poblacional específico, en un ámbito concreto de organizaciones, dentro del contexto geográfico e institucional de Andalucía (España). Los hallazgos de esta investigación están orientados a una perspectiva aplicada, de forma que puedan guiar propuestas y sugerencias en el ámbito de la educación para la ciudadanía digital de las personas jóvenes andaluzas.

El acceso y empleo de los recursos digitales se ha convertido en una poderosa herramienta de participación en manos de la ciudadanía, aunque el aprovechamiento de los mismos continúa siendo desigual. En España, y en Andalucía, encontramos evidencias de que las desigualdades de acceso o primera brecha digital están relativamente superadas, pero persisten diferencias en cuanto a las competencias digitales más avanzadas (Fernández Prados et al, 2020). No debemos olvidar que el modo de empleo de los recursos digitales condiciona las oportunidades de participación y los potenciales beneficios aparejadas a su uso. Los datos muestran que el uso de redes sociales con fines participativos o socialmente transformadores sigue siendo modesto entre las personas jóvenes andaluzas (Fernández Prados et al., 2020).

Todo ello nos lleva a preguntarnos por los factores contextuales y sociales que inciden en la intensidad y en las formas de la participación digital de éstos. Dicha participación puede materializarse en un heterogéneo abanico de prácticas activistas (Fernández Prados y Lozano Díaz, 2021), y en muchos casos supone la interacción con organizaciones de la sociedad civil, que actúan canalizando las demandas de la ciudadanía. Por tanto, la participación digital no se limitaría al uso de los medios online para las relaciones -más o menos directas- entre los ciudadanos y la administración o el poder político. Hemos de prestar también atención al involucramiento en entidades interlocutoras que funcionan como contrapeso de dicho poder y dialogan con él (Putnam, 1993, Almond y Verba, 1963). Dentro del amplio conjunto de organizaciones posibles a analizar, elegimos centrarnos en las Entidades del Tercer Sector de Acción Social, continuando la labor de algunos estudios sobre la cuestión en Andalucía, y tratando de cubrir una laguna en cuanto a la participación específicamente juvenil (Rodriguez y Marbán, 2015, Caralt et al, 2017, Candón-Mena, 2018, Arrieta et al, 2019).

\section{Objetivos}

Esta investigación usa metodología cualitativa para examinar la interrelación entre (a) las estrategias y herramientas que las organizaciones del Tercer Sector de Acción Social utilizan para difundir sus mensajes en la red y atraer a miembros y simpatizantes, con especial atención a las prácticas empleadas para acercarse a los jóvenes, y (b) las actitudes hacia la participación de jóvenes por medios digitales, con énfasis en los discursos relativos a las organizaciones del Tercer Sector de Acción Social.

Desde un enfoque interaccionista, entendemos que la participación no puede analizarse únicamente a través de las características (individuales o sociales) de los sujetos potencialmente activistas. Esta participación depende igualmente de la oferta disponible: tanto la administración como los distintos sectores del activismo organizado median las oportunidades existentes para una participación efectiva. 
En el apartado de resultados adelantamos algunos hallazgos preliminares acerca de los elementos que obstaculizan la implicación digital de jóvenes en las entidades del Tercer Sector de Acción Social.

\section{Metodología}

La perspectiva de investigación parte del análisis de un fenómeno relacional, examinando actitudes y prácticas de las dos partes involucradas: personas jóvenes andaluzas y entidades del Tercer Sector de Acción Social en la misma comunidad autónoma. Tratamos de investigar, por un lado, el funcionamiento de la oferta -oportunidades participativas que genera la configuración institucional de las entidades analizadas-, por otro lado, el funcionamiento de la demanda -disposiciones participativas de personas jóvenes y la valoración que hacen de las medidas puestas en práctica para atraer su compromiso-. Los participantes (tanto en el caso de las entrevistas como en el de los grupos de discusión) fueron contactados mediante redes personales. El diseño de la investigación prevé la realización de unas 15-20 entrevistas y 3 grupos de discusión, aunque el trabajo de campo se encuentra actualmente en curso.

Para el análisis de la oferta, recurrimos al uso de entrevistas abiertas semiestructuradas con responsables de comunicación y gestión de redes sociales de diversas entidades del Tercer Sector de Acción Social. Para delimitar la muestra de análisis procedimos a un muestreo no probabilístico de tipo intencional, seleccionando aquellas organizaciones pertenecientes a la Plataforma de ONG de Acción Social (https://www.plataformaong.org/). Esta plataforma aglutina a las entidades más representativas del sector en la región, como EAPN, Cruz Roja Andalucía o la Plataforma Andaluza del Voluntariado. El recurso a las entrevistas abiertas se justifica en los siguientes puntos:

Primero, constituyen una excelente herramienta para recoger en profundidad el discurso de actores que ocupan posiciones clave en los fenómenos objeto de análisis -en este caso, responsables de las estrategias de comunicación que pueden incidir en las actitudes participativas de la ciudadanía-. Además, las prácticas de comunicación digital de las entidades constituyen un fenómeno dinámico, que ha de ser examinado atendiendo a su evolución a lo largo del tiempo y a los factores que explican dicha evolución. Bien realizadas, las entrevistas en profundidad proporcionan información secuencial y facilitan la exposición de hechos cronológicamente ordenados, permitiendo al investigador reconstruir estas "cadenas de contingencias" (Becker, 2010, pp. 54-55).

Segundo, las entrevistas semiestructuradas permiten captar simultáneamente las estrategias (en calidad de representantes de las entidades analizadas) y los argumentarios que envuelven dichas estrategias. El discurso recogido en situación de entrevista se encuentra en un nivel intermedio entre el orden del decir y el orden del hacer (Alonso, 1999), lo que permite acceder tanto a las prácticas como a las categorías simbólicas desde las que se interpretan. Las estrategias comunicativas y participativas de las entidades están atravesadas por diversas creencias sobre lo que es necesario o superfluo, urgente o aplazable, conveniente o inconveniente.

Tercero, las entidades del Tercer Sector de Acción Social constituyen un grupo heterogéneo de organizaciones, desiguales en cuanto a sus recursos, tamaño, antigüedad y estructura organizativa. Partimos del supuesto de que estos elementos articulan diversas formas de poner en marcha su oferta participativa. Las entrevistas semiestructuradas -una vez acumulado un número suficiente de ellas- nos permiten confrontar sistemáticamente distintos discursos, obtenidos de sujetos que actúan en contextos institucionales muy diversos. Estos análisis son útiles para establecer mecanismos explicativos de la relación entre orientaciones práctico-discursivas de los responsables y características de las entidades (Small, 2009). 
El guion de las entrevistas se planteó de forma flexible, aunque se trató de integrar los siguientes temas: proceso de digitalización de la entidad, presencia de la entidad en internet y redes sociales y gestión de las mismas, estrategias de comunicación de la entidad, estrategias de fomento de participación por parte de la entidad (oportunidades que se ofrecen para influir en la actividad de la entidad, medidas tomadas para lograr ampliar su base e implicar a la ciudadanía) y estrategias específicas para la comunicación y la participación con las personas jóvenes. Para elaborar el guion y conducir la entrevista nos basamos en la propuesta de Sanz (2017), que adapta el concepto de Escalera de Participación de Arnstein a la web 2.0. Así, comenzamos con preguntas referentes a los canales de comunicación desde dentro de la entidad hacia fuera (difusión e información) y tratamos de ir avanzando hacia estrategias centradas en la participación desde fuera hacia dentro (colaboración y participación).

Para analizar la demanda, recurrimos al uso de grupos de discusión, compuestos por andaluces menores de 30 años. Seleccionamos jóvenes de ambos sexos, bajo el criterio de tener una actividad continuada en la red y experiencias de uso de las redes sociales (teorizamos que una selección aleatoria podría llevarnos a sujetos muy distanciados del medio digital, sin discursos relevantes sobre la participación online). Elegimos la técnica del grupo de discusión para recoger las representaciones colectivas más extendidas en el grupo social objeto de estudio, y para captarlas en su forma cotidiana, tal como es utilizada entre los grupos de iguales. Partimos del grupo de discusión como herramienta de investigación que produce los discursos sobre la cuestión puestos asiduamente en juego frente a los próximos sociales, donde el desarrollo de la interacción va laminando progresivamente la censura estructural (Martín Criado, 1997).

En el marco de una investigación cualitativa, la aproximación realizada incluye el uso de varias estrategias a la hora de analizar un mismo fenómeno, por ejemplo, el uso de varios métodos (entrevistas individuales y grupos de discusión en el caso presente de estudio). De este modo, se cree que los puntos débiles de cada herramienta concreta no se solapan con los de las demás y que, por el contrario, sus puntos fuertes se suman. Al utilizar una sola técnica, los estudios son más vulnerables a los sesgos y fallos metodológicos inherentes a cada una de ellas y que la articulación y combinación de herramientas ofrece la alternativa de poder visualizar un problema desde diferentes perspectivas y miradas, y de esta manera aumentar la consistencia de los hallazgos (Valles, 1999, Patton, 2015).

\section{Resultados}

Tratándose de una investigación en curso, los resultados aquí expuestos han de entenderse como preliminares y a la espera de un mayor desarrollo del trabajo de campo. De los hallazgos que se desprenden del análisis del material, nos centraremos aquí en los elementos detectados que dificultan una estrategia de comunicación eficaz y obstaculizan una plena implicación digital de las personas jóvenes andaluzas en las entidades analizadas. Estos elementos incluyen:

- En el seno de las entidades, existe una clara prioridad hacia la acción sobre el terreno y la atención a los colectivos usuarios. Esto conlleva dejar el papel de la actividad digital en un segundo plano ante la escasez de medios materiales y humanos. Este hecho se agrava por el peso del trabajo voluntario en el funcionamiento interno de las organizaciones, donde sólo a veces encontramos personal asalariado especializado en la gestión de la comunicación online. En estos casos, las estrategias de fomento de la participación por vías online muestran altos grados de amateurismo y espontaneísmo. Las entidades tienden a percibir la comunicación digital como un elemento separado de su actividad cotidiana, limitada a la labor de sus responsables -expertos o no-, que no requiere de la implicación colectiva de la organización para su correcto funcionamiento.

- Carácter descentralizado de muchas de las entidades, que se proyecta en su presencia digital produciendo un mensaje fragmentado: en muchos casos no hay una identidad online reconocible que transmita un discurso unificado en las distintas redes sociales. 
La actividad online de las entidades tiende a fraccionarse en multitud de cuentas y webs (asociadas a proyectos en marcha, a secciones subregionales de la entidad o a asociaciones federadas con sus propios mensajes y simbologías), sin proyección de una imagen coherente. Esta descentralización también supone que importantes labores de comunicación y contacto recaen, en muchos casos, en grupos locales con pocos recursos y medios para la actividad digital.

- Dificultad para atraer de forma estable a profesionales especializados que reúnan, por un lado, competencias de comunicación y habilidades técnicas de gestión informática, y por otro, cierta implicación con la causa de la entidad y conocimiento de la red asociativa y su funcionamiento. Estos perfiles profesionales disfrutan de una demanda creciente en el mercado de trabajo y son atraídos en muchos casos por empresas tradicionales del sector privado.

- Competencia difícil en un mercado de proyectos activistas donde las entidades del Tercer Sector son poco atractivas para su público potencial (jóvenes de perfil activista). Muchas entidades se ven lastradas por una imagen excesivamente institucional, burocratizada y sistémica, percibidas como parte del "mundo adulto". En este sentido, son eclipsadas por otras iniciativas militantes desarrolladas en los márgenes, con retóricas contraculturales y funcionamiento informal.

- Para muchos jóvenes, los procesos de "politización" o toma de conciencia vía red comienza muchas veces a partir de experiencias personales: problemas en el ámbito laboral, estudiantil, habitacional o afectivo-sexual (p.ej: acoso o agresiones en casos de mujeres y/o LGTB). Estas experiencias, al ser compartidas con otros usuarios en situaciones similares, retroalimentan la disposición al activismo y la protesta social. Ello lleva al contacto y seguimiento prioritario con organizaciones representativas de su colectivo o que canalizan demandas que les afectan personalmente. Las entidades del Tercer Sector son percibidas como centradas en segmentos de la población en situaciones de vulnerabilidad extrema, con un tipo de participación dirigida a prestar asistencia a otros, pero no para solucionar problemas propios.

\section{Conclusiones}

Las dinámicas de participación juvenil constituyen un fenómeno interactivo: su configuración e intensidad depende de la acción de dos partes, jóvenes activistas y organizaciones que canalizan su implicación. En este trabajo hacemos uso de varios métodos cualitativos para examinar ambos lados de la relación y fortalecer los hallazgos a los que accedemos con cada técnica de investigación. Este tipo de aproximación resulta especialmente relevante para captar las relaciones entre organizaciones de cualquier tipo con su público actual o potencial. Además, la investigación pone de manifiesto la necesidad de adaptar las herramientas cualitativas en función del tipo de informante. En nuestro caso, el grupo de discusión es utilizado para producir un discurso -informal y cotidiano- representativo de un grupo social amplio (el de las personas jóvenes ciberactivistas). Mientras la entrevista semiestructurada nos permite acercarnos a relatos con mayor detalle técnico y donde el orden temporal de los acontecimientos es particularmente relevante (los discursos de responsables de comunicación de las entidades).

Este diseño nos permite observar que los obstáculos a superar existen a ambos lados de la relación. Aunque nuestros resultados son preliminares, detectamos que las organizaciones del tercer sector son conscientes de sus limitaciones y tratan de superarlas, pero carecen en muchos casos de los medios para hacerlo y/o arrastran configuraciones institucionales difícilmente reversibles. Esta investigación pone en evidencia que mejorar las estrategias de comunicación y participación -especialmente, las dirigidas a las personas jóvenes- requiere de transformaciones amplias en la estructura organizativa de las entidades. Este tipo de transformaciones necesitan del compromiso de la organización en su conjunto, no pudiendo ser solventadas por los departamentos y responsables de comunicación en solitario. 


\section{Agradecimientos}

Este trabajo se enmarca en el proyecto "Ciudadanía digital entre los jóvenes andaluces: claves para el ciberactivismo y los cibermovimientos sociales en Andalucía" (PRY109/19) financiado por la Fundación Centro de Estudios Andaluces.

\section{Referencias}

Almond, Gabriel A., \& Sidney Verba. (1963). The Civic Culture. Political Attitudes and Democracy in Five Nations. Newbury Parke: Sage.

Alonso, L. E. (1999). Sujeto y discurso: el lugar de la entrevista abierta en las prácticas de la sociología cualitativa. En J. M. Delgado y J. Gutiérrez (eds). Métodos y técnicas cualitativas de investigación en ciencias sociales: 225-249. Madrid: Síntesis.

Arrieta, E., De Haro, F., \& Zafra, J. M. (2019). Informe - impacto digital en las fundaciones. Revolución digital en lo social. Madrid: Asociación Española de Fundaciones.

Becker, H. (2010). Trucos del oficio. Madrid: Siglo XXI.

Candón-Mena, J. (2018). Actas del Il Congreso Internacional Move.net sobre Movimientos Sociales y TIC. Sevilla: COMPOLÍTICAS

Caralt, E., Carreras, I., y Sureda, M. (2017). La transformación digital en las ONG. Conceptos, soluciones. Madrid: ESADE

Castells, M. (2012). Redes de indignación y esperanza: los movimientos sociales en la era de Internet. Madrid: Alianza Editorial

Fernández Prados, J.S. \& Lozano Díaz, A. (2021) El reto de la ciudadanía digital activa en la educación superior europea: análisis del ciberactivismo entre los estudiantes universitarios. EDMETIC, 10(1), 118-134.

Fernández Prados, J.S., Robles Morales, J.M. \& Guevara Gil, J.A. (2020). Nuevas y viejas brechas digitales entre los jóvenes. El reto de la participación digital. Comunicación en el X Congreso Andaluz de Sociología (Jaén).

Martín Criado, E. (1997). El grupo de discusión como situación social. Revista Española de Investigaciones Sociológicas, 79, 81-112.

Ortiz, R. (2016). Social cybermovements: A review of the concept and theoretical framework. Communication and Society, 29(4), 165-182

Patton, M. Q. (2015). Qualitative research and evaluation methods. Thousand Oaks: SAGE Publications.

Putnam, R. (1993). Making democracy work: Civic traditions in modern Italy. Pricenton: Pricenton Univesrity Press.

Rodríguez, G., y Marbán, V. (2015). Análisis prospectivo sobre los retos actuales y futuros del Tercer Sector de Acción social. Madrid: POAS

Sanz, R. M. (2017). La evaluación de la participación en sitios web: una propuesta metodológica aplicada a museos. Estudios Sobre El Mensaje Periodístico, 23(2), 1229-1246

Small, M. L. (2009). How many cases do I need? On science and the logic of case selection in fieldbased research. Ethnography, 10(1), 5-38.

Valles, M. S. (1999). Técnicas cualitativas de investigación social. Madrid: Síntesis. 